\title{
Equivalent Lateral Spacing Operation to Improve Airspace Efficiency of V-opening Runway at Chengdu New Airport
}

\author{
Kun $\mathrm{Cao}^{1}$, Zhiyuan Shen ${ }^{2, *}$ and Chaoyang $\mathrm{Lu}^{2}$ \\ ${ }^{1}$ Southwest Air Traffic Management Bureau CAAC, Chengdu, China \\ ${ }^{2}$ College of Civil Aviation, Nanjing University of Aeronautics and Astronautics, Nanjing, China \\ ${ }^{*}$ Corresponding author
}

\begin{abstract}
In recent years, with the rapid development of civil aviation industry, new airport is necessary to plan and construct to the increasing demand for civil aviation. Current parallel runway instrument approach regulations require that divergence angle established between two go-around tracks of independent parallel approach must be larger than 30 degrees. Restricted by the above regulations, Chengdu New Airport runway configuration is with $\mathrm{V}$-opening runway which not only causes the waste of airspace resources, but also causes various difficulties in actual operation. In this paper the equivalent lateral spacing operation (ELSO) is used to V-opening runway to improve airspace efficiency. Firstly, the paper analyzes the drawbacks of Chengdu new airport operation based on the current regulations. Secondly, the concept of ELSO is introduced to establish the divergence angle model of the parallel runway. The ELSO mode at Hartsfield-Jackson Atlanta International Airport (ATL) is summarized. Finally, based on the runway configuration of Chengdu New airport, the improvement of airspace efficiency under ELSO is given.
\end{abstract}

Keywords-independent parallel approach; runway capacity; equivalent lateral spacing; operation; V-opening runway

\section{INTRODUCTION}

In recent years, China's civil aviation industry has developed rapidly. According to statistics, from 2012 to 2016, the number of passenger traffic in china civil aviation industry increased from 319 million to 488 million, up to $52.9 \%$; the number of airport freight traffic increased from 545.0 million tons to 668.0 million tons, up to $22.6 \%$; the number of airport movements increased from 545.0 million tons to 668.0 million tons, up to $22.6 \%$. Chengdu, as a major economic center in western China and a center of commercial and financial logistics in China, has maintained a high level of civil aviation business in the past decade. Statistically, from 2000 to 2014, the average annual growth rate of air passenger throughput in Chengdu area reached $14.7 \%$, and will maintain a rapid development trend in the future.

The passenger throughput in Chengdu is expected to reach 75 million in 2025. However, due to some restriction like tight airspace and closer distance to Chengdu city, Chengdu Shuangliu airport has no capacity for expansion in the absence of total airport. Therefore, it is necessary to plan and build Chengdu new airport. Chengdu airport runway layout geometries as shown below.

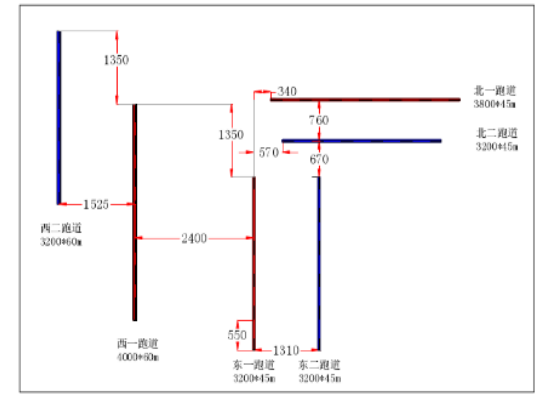

FIGURE I.CHENGDU NEW AIRPORT RUNWAY CONFIGURATIONS

Chengdu new airport runway layout geometries consists of 6 runways, three red runways are the first-stage project, and three blue runways are the second phase project. It consists of a set of two wide spaced parallel runways in the west of flight area (meet independent approach), a set of two runways (meet independent approach) in the southeast and a set of two lateral, mid-range parallel runways in the northeast of flight area, in which the lateral runway is used for eastward departure, and four parallel runways will be taken off and arrived at a different demand pattern, belonging to a typical V-opening runway.

According to Regulation of Parallel Runway Simultaneous Instrument Operation, the requirement of conducting independent parallel departure procedures at the airport with multiple parallel runways is the course between two departure paths must diverge by 15 degrees (deg) or more immediately after departure; the requirement of conducting relevance /parallel approach procedures is the course between two goaround paths or the go-around path and the departure path of adjacent runway must diverge by 30 degrees (deg) or more. There are following drawbacks pertaining to such procedures aiming at Chengdu new airport V-opening runway: 1. Limited capacity growth in the current situation of terminal airspace tension;2. In the case of three runways simultaneously operating in the current planning, if the East-1 runway goaround to the right to offset by 15 degrees(deg), the track will overlap with the North-1 runway, thus interfering with the normal operation of the North-1 runway. 3. In the forward planning runway operation condition, if the East-1 runway goaround to the right to offset by 30 degrees(deg), it will interfere with the normal operation of the North-1 and North-2 runways.4. In the long-term planning, the number of southnorth parallel runways will reach four, which will cause 
difficulties in actual operation Therefore, it is necessary to find the appropriate method to solve the original departure / goaround path divergence angle problem, and then solve the above problems.

In recent years, extensive implementations of Area Navigation (RNAV) Standard Instrument Departure (SID) procedures that result in improved aircraft navigational precision give cause to design more advanced and efficient terminal area flight procedures. Under the precondition of ensuring the departure track of the parallel runway without violating the divergence, it is worth studying to reduce the current 15-degree divergence angle to use the terminal airspace more efficiently and realize the airport capacity upgrading.

In the above context, Equivalent Lateral Spacing Operations(ELSO) standard was proposed, and FAA conducted a one-year implementation at The Hartsfield-Jackson Atlanta International Airport (ATL).

The benefits of ELSO are: Programmers can be more flexible layout of the departure, go-around paths; air traffic controllers are able to release more aircraft with a smaller divergence angle; simultaneously, it will increase the capacity of busy multi-runways airport greatly and reduce flight delays while meeting the airport noise reduction requirements maximum in restricted .

However, the ATL application case improves the airspace utilization rate, on the one hand it only applies to the parallel runways, on the other hand, it does not give the capacity enhancement possible value. Therefore, Therefore, this paper takes Chengdu Airport as an example to study the operation scheme of Equivalent Lateral Spacing Operations(ELSO) in Vopening runway.

\section{EQUiVALENT LATERAL SPACING OPERATION}

\section{A. The ELSO Concept}

The core concept of ELSO is : the lateral spacing of independent parallel departures path after reducing the divergence angle can reach the minimum of that under the standard of divergence angle is not less than 15 degree, in other word, the lateral spacing of the departing aircraft in both cases is equivalent. The improvement of airport capacity by using the departure/go-around procedure based on the equivalent lateral spacing operations(ELSO) standard is achieved mainly by the following methods: By reducing the Conventional standard of the 15 degrees departure divergence angle and the 30 degrees go-around divergence angle, can be increased the departure/goaround path, which achieving more efficient use of airspace; By changing the original divergence angle and the original path can be reduce the influence between runways, which improving the capacity of the airport.

The ELSO standard concept for diverging departure operations takes into consideration and capitalizes on three factors: parallel departure runway spacing, parallel departure runway stagger, departure path following characteristics. By rationally utilizing the runway configuration of the airport, it is theoretically possible to achieve the 5-10 degrees reduction of the track divergence angle of independent departure aircraft between parallel. To obtain a suitable divergence angle for a specific runways configuration, it is necessary to study the current control operation method that is, the lateral spacing between the conventional operations, 15 degrees or 30 degrees divergence angle to departure or go-around, as the basis of determining the standard of the equivalent lateral spacing.

\section{B. Independent Parallel Departure Model Based on Equivalent Lateral Spacing Operation}

In order that the lateral spacing between the departure path of independent parallel departures runway and the ELSO divergence angle departure path at a certain distance from the runway ends, can achieve even better than that when the same departure run is performed at a 15 degrees divergence angle. Therefore, it is first necessary to study the lateral spacing achieved by the flight procedure at the traditional 15-degree divergence angle and use it as the baseline spacing for the parallel independent departure procedure based on ELSO.

Independent parallel departure operations standard requires a minimum parallel runway spacing $r_{\min }$ of $760 \mathrm{~m}$ and the assignment of headings representing nominal departure courses that differ in course angle $\alpha_{\min }$ by a minimum of 15 deg. as the minimum parallel runway spacing,as the minimum diverging angle. As shown below, dashed lines represent nominal departure or go-around tracks.

Based on the runway layout geometry, the spacing between the nominal departure tracks depends on the distance $\mathrm{d}$ from the runway end and is denoted as nominal spacing $n(d)$. The nominal spacing is given

$$
n(d)=r_{\min }+d * \tan \left(\alpha_{\min }\right)
$$

Due to the influence of aircraft performance, wind and other factors, the flight path of actual go-around or departure aircraft will be slightly biased nominal flight path, so, the yellow and red shadow areas indicate the divergence of actual departure path. The width of the departure path that closely follows the extended center line of a runway is characterized by $\sigma_{\mathrm{sto,c}}$.The sto,c is used here to denote Straight-out Conventional departure operations. Similarly, $\sigma_{\mathrm{DIV}, \mathrm{C}}$ characterizes the width of the departure path whose center line is angled relative to the runway center line and the subscript DIV,C denotes Diverging Conventional departure operations.

$$
s(d)=r_{\min }+d^{*} \tan \left(\alpha_{\min }-\sigma_{D I V, C}\right)-\mathrm{d}^{*} \tan \left(\sigma_{S T O, C}\right)
$$

This spacing can be viewed as the minimum lateral spacing between the actual departure paths authorized by the currently 30-deg divergence angle departure operation standard, and will serves as the baseline spacing for all ELSO standard application requirements.

When the spacing of parallel runways exceeds $760 \mathrm{~m}$, to some extent, it will cause the waste of airspace resources if still adopt the not less than 15-deg divergence angle to implement independent parallel departure operations. Therefore, according to the runway layout geometry, departure tracking divergence angle between parallel runways can be reduced appropriately. In addition, with the promotion of the RNAV departure operations, navigational precision in terminal airspace can be 
greatly improved, to a certain extent, divergence angle between departure aircraft can be reduced, i.e., in the RNAV operations, $\sigma_{\mathrm{STO}, \mathrm{C}}, \sigma_{\mathrm{DIV}, \mathrm{C}}$ will be reduced as indicated in the Figure. 2.

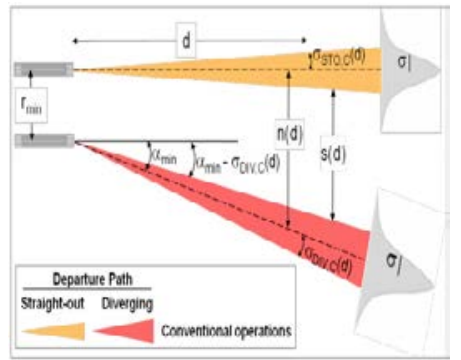

FIGURE II.

PARALLEL RUNWAY DIVERGENCE ANGLE DEPARTURE OPERATIONS

Therefore, in this case, it is feasible to meet the baseline spacing requirements with conducting independent parallel departure operations with reduced divergence angle. The above figure illustrates the basic situation diverging application of the ELSO standard to RNAV departure operations. Given the spacing baseline, the widths of the departure paths, and the runway stagger $t$, the runway spacing is given by:

$$
\mathrm{r}=\mathrm{s}(\mathrm{d})+\mathrm{d} * \tan \left(\sigma_{\mathrm{STO}, \mathrm{R}}(\mathrm{d})\right)-(\mathrm{d}+\mathrm{t}) \tan \left(\beta-\sigma_{\mathrm{DIV}, \mathrm{C}}(\mathrm{d}+\mathrm{t})\right)
$$

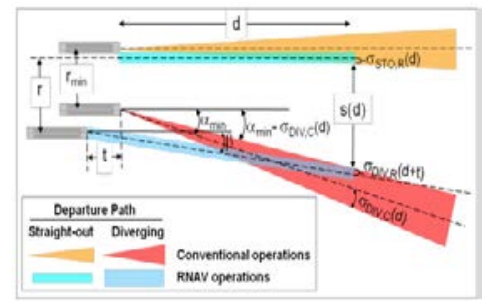

FIGURE III.

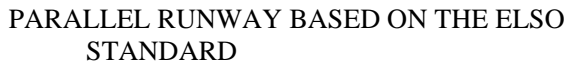

The width of the departure path that closely follows the extended center line of a runway is characterized by $\sigma_{\mathrm{STO}, \mathrm{R}}$. The subscript STO,R is used here to denote Straight-out Conventional departure operations. Similarly, $\sigma_{\text {DIV,R }}$ characterizes the width of the departure path whose center line is angled relative to the runway center line and the subscript DIV,R denotes Diverging Conventional departure operations. Therefore, based on the specific runway configurations, i.e., given the parallel runway spacing, and the runway stagger, the diverging application of the ELSO standard enables a reduced divergence angle $\beta$. is given by

$$
\beta=\arctan \left\{\left(\frac{1}{d+t}\right) *\left(s(d) * \tan \left(\sigma_{\mathrm{STO}, \mathrm{R}}(\mathrm{d})\right)\right)\right\}+\sigma_{\mathrm{DIV}, \mathrm{R}}(\mathrm{d}+\mathrm{t})
$$

The reduced divergence angle is defined to provide, at distance $d$, spacing of the departure paths that is equivalent to the baseline spacing. In fact, when parallel runway spacing is greater than $760 \mathrm{~m}$ and at shorter distances, the spacing of the diverging departure paths exceeds the baseline spacing.

\section{Mode at The Atlanta International Airport (ATL)}

Atlanta International Airport(ATL) is one of the busiest airports in the world, with 857,000 take-off and landing aircraft in 2014 and 2348 sorties per daily. The airport has five EastWest parallel runways, of which two runways (8L / 26R and 9R / 27L) serve as the primary approach runways, two runways (8R / 26L and 9L / 27R) serve as the primary departure runways, the fifth runway $(10 / 28)$ is provided as required for approach or departure. After the application of the ELSO standards, it can better accommodate airspace and increase the forth departure path. As indicated in Figure.4.

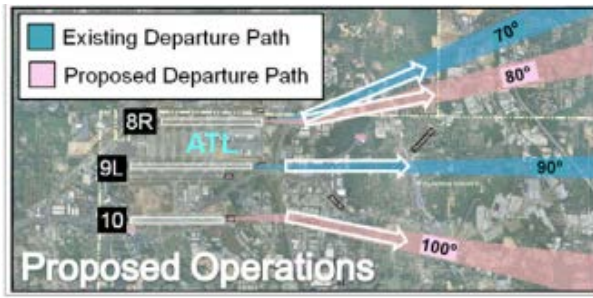

FIGURE IV.

DEPARTURE PATHS OF ELSO IN ATL

\section{OPERATION MODE OF V-OPENING RUNWAY BASED ON ELSO}

According to China Civil Aviation Regulations, independent parallel approach departures operations from parallel runways, the course between departure path and adjacent runway approach go-around path must diverge by 30 degrees (deg) or more immediately after departure;the course between go-around path and adjacent runway approach goaround path must diverge by 30 degrees (deg) or more. From the above analysis, the parallel runway spacing is greater than 760 meters, it will also cause the waste of airspace resources and other adverse effect if Implementation independent parallel approach operation with the divergence angle diverging 30 degrees or more.

Taking a new airport as an example, an independent parallel approach model based on equivalent lateral spacing is established. The airport three runways configuration as shown below.

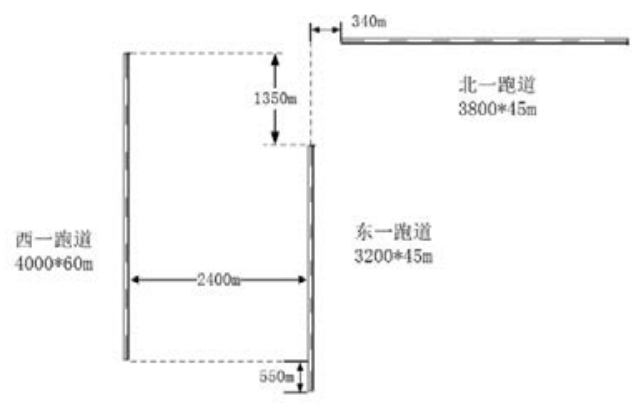

FIGURE V. AIRPORT RUNWAY LAYOUT GEOMETRIES

This period plans three runways at Chengdu new airport, the North-1 runway only for the East departure, the East-1 runway, and West-1 runway can be used for North arrival/departure. 
The basic ILS surfaces assessment and the OAS surfaces assessment of the East-1 runway and the North-1 runway show that the missed approach surface of East-1 runway will cover the North-1 runway, i.e., The East-1 go-around aircraft will affect the North-1 runway Conducting the conventional 30-deg divergence departure operations, which has a great impact on the runway capacity of the airport. Therefore, it is possible to consider the reduction of the divergence angle of the East- 1 runway by the independent parallel approach based on the ELSO through the West-1 runway and East-1 runway to reduce the effect of the East-1 aircraft on the North-1 runway.

When the parallel runway spacing is the minimum distance required for the implementation of the independent parallel approach operation (1035 m), the go-around aircraft is flown to the head of the runway $10.4 \mathrm{~km}$ when the 30 -degree divergence angle is maintained in the traditional go-around procedures., with the adjacent runway off the departure aircraft can establish $6 \mathrm{~km}$ lateral spacing, so the scope of the Chengdu New Airport East-1 runway implementation of ELSO go-around standard feasibility analysis within the West runway $10.4 \mathrm{~km}$ (East runway $11.8 \mathrm{~km})$.

\section{A. Relevant operation of West-1 and East-1}

When the spacing of the parallel runways is the minimum spacing required to implement independent parallel approach, $915 \mathrm{~m}$. As indicated in the Equation (2), the goaround aircraft is flown to the head of the runway $10.4 \mathrm{~km}$ when the 30-degree divergence angle is maintained in the traditional go-around procedures., with the adjacent runway off the departure aircraft can establish $6 \mathrm{~km}$ lateral spacing, so the scope of the Chengdu New Airport East-1 runway implementation of ELSO go-around standard feasibility analysis within the West runway $10.4 \mathrm{~km}$ (East runway $11.8 \mathrm{~km}$ ).

In the implementation of the ELSO standard conditions, the East-1 go-around aircraft can guarantee a minimum of 5.5km.i.e., East-1 departure /go-around aircraft (North Run) at latest at the distances up to $5.5 \mathrm{~km}$ from the runway ends. The lateral spacing obtained at the same distance by the Straight-out go-around procedure before $5.5 \mathrm{~km}$ is not less than the lateral spacing obtained by performing the 30 -degree divergence angle. on the other hand, the configuration and location of East-1 runway and North-1 runway shows that, the angle between the wire defined by the point take-off end of the East-1 runway and North-1 runway threshold and the north extended line of the East-1 runway is 13.3 degrees. i.e., The divergence angle of the East-1 departure/go-around aircraft ((North Run) to the right is not greater than the angle in that case, which can guarantee the normal operation of the North-1 runway.

Based on the above analysis, to meet the equivalent lateral operation (ELSO)conditions, to meet the equivalent lateral interval operating(ELSO) conditions, when the East-1, West-1 runway according to the relevant parallel approach operations, give the following two kinds of suggestions:

(1)East-1 runway overshoot aircraft fly over the intersection of the East-1 and the north extended line of the East-1 runway, which is then offset to the right after the first runway $1430 \mathrm{~m}$.

Calculate that the aircraft bending go-around radius is $1.22 \mathrm{~km}$, and the distance between the nominal track and the reference track (i.e., the red part in Figure 7) is 1373m; the most recent distance between the outer boundary of the nominal track turning area and the reference track is $809 \mathrm{~m}$. That is, its turn land does not overlap with its reference track, the East-1 runway by the equivalent lateral spacing operation(ELSO) standard go-around, can protect the actual distance between go-around or departure aircraft from West-1, East-1 runway is always better than its reference spacing.

(2) Reduce the divergence angle. of the East-1 runway departure / go-around aircraft in the north when running.

Calculate that the right angle of aircraft can not be less than 6.3 degrees (Otherwise it will not be able to achieve the establishment of $6 \mathrm{~km}$ lateral spacing under the premise that the actual spacing between the go-around or departure aircraft from the West- 1 and the East-1 runway is always better than its reference spacing); and it can not be greater than 13.3 degrees (Otherwise, the East-1 runway departure/go-around aircraft will affect the normal operation of the North-1 runway).

It is recommended that the divergence angle of the East-1 departure / go-around aircraft be reduced to 10 degrees.

\section{B. Independent operation of West-1 and East-1}

When the spacing of the parallel runways is the minimum spacing required to implement independent parallel approach, $1035 \mathrm{~m}$. With the above analysis, the scope of the Chengdu New Airport East-1 runway implementation of ELSO go-around standard feasibility analysis within the West runway $10.4 \mathrm{~km}$ (East runway 11.8km).

In the implementation of the ELSO standard conditions, the East-1 go-around aircraft can guarantee a minimum of $5.1 \mathrm{~km}$. i.e., aircraft at latest at the distances up to $51 \mathrm{~km}$ from the runway ends. The lateral spacing obtained at the same distance by the Straight-out go-around procedure before $5.1 \mathrm{~km}$ is not less than the lateral spacing obtained by performing the 30 degree divergence angle. On the other hand, the configuration and location of East-1 runway and North-1 runway shows that, the angle between the wire defined by the point take-off end of the East-1 runway and North-1 runway threshold and the north extended line of the East-1 runway is 13.3 degrees. i.e., The divergence angle of the East-1 departure/go-around aircraft ((North Run) to the right is not greater than the angle in that case, which can guarantee the normal operation of the North-1 runway.

Based on the above analysis, to meet the equivalent lateral operation (ELSO)conditions, to meet the equivalent lateral interval operating(ELSO) conditions, when the East-1, West-1 runway according to the independent parallel approach operations, give the following two kinds of suggestions:

(1)East-1 runway overshoot aircraft fly over the intersection of the East- 1 and the north extended line of the East-1 runway, which is then offset to the right after the first runway $1430 \mathrm{~m}$.

Calculate that the aircraft bending go-around radius is $1.22 \mathrm{~km}$, and the distance between the nominal track and the reference track (i.e., the red part in Figure7) is 1257m; the most recent distance between the outer boundary of the nominal track turning area and the reference track is $694 \mathrm{~m}$. That is, its turn land does not overlap with its reference track, the East-1 
runway by the equivalent lateral spacing operation(ELSO) standard go-around, can protect the actual distance between goaround or departure aircraft from West-1, East-1 runway is always better than its reference spacing.

(2) Reduce the divergence angle of the East-1 runway departure / go-around aircraft in the north when running.

Calculate that the right angle of aircraft can not be less than 6.9 degrees (Otherwise it will not be able to achieve the establishment of $6 \mathrm{~km}$ lateral spacing under the premise that the actual spacing between the go-around or departure aircraft from the West-1 and the East-1 runway is always better than its reference spacing); and it can not be greater than 13.3 degrees (Otherwise, the East-1 runway departure/go-around aircraft will affect the normal operation of the North-1 runway).

It is recommended that the divergence angle of the East-1 departure / go-around aircraft be reduced to 10 degrees.

\section{CONCLUSION}

This paper studies the operation mode of $\mathrm{V}$-opening runway based on equivalent lateral spacing operations(ELSO). First, taking Chengdu New Airport as an example, the paper analyzes the drawbacks of Chengdu new airport operation based on the current regulations. Secondly, the concept of ELSO is introduced, and the divergence angle model of the parallel runway is established. Taking the Atlanta International Airport (ATL) as an example, the advantages of the operation mode are summarized, and then the independent parallel approach operation model of $\mathrm{V}$-opening runway based on equivalent lateral spacing is established. Finally, based on the runway configuration of Chengdu New airport, the operation scheme of Chengdu New airport based on the effective lateral interval is given. The results of this study show the feasibility of the relevant / independent parallel mode based on the equivalent lateral spacing operations(ELSO) in the V-opening runways, which provides theoretical support for the operation of the $\mathrm{V}$ opening runway in China.

\section{ACKNOWLEDGEMENT}

The authors would like to thank the financial support by National Natural Science Foundation of China (No.61501225).

\section{REFERENCES}

[1] HARRIS R M.Models for runway capacity analysis[R].Washington DC:Mitre Corporation,1969,40 55.

[2] R.S.Ratner. A methodology for evaluating the capacity of air traffic control systems [R].FAA_RD_70_69, 1970: 1-175.

[3] NEWWELL G F.Airport capacity and delays[J].Transportation Science,1979,13(3): 200 250

[4] Bertsim as,D.and S.Stock.The Air Traffic Flow Management Problem with Enroute Capacities[R].Operations Research,1998,46:400 430

[5] Peter C. Kuzminski.AN IMPROVED RUNWAY SIMULATOR SIMULATION FOR RUNWAY[C]. Integrated Communications, Navigation \& Surveillance Conference ,2013, 5 (5) :1-11.

[6] RalfH.Mayer, DennisJ.Zondervan , AlbertA.Herndon , TylerSmith A Standard for Equivalent Lateral Spacing Operations - Parallel and Reduced Divergence Departures[R]. Ninth USA/Europe Air Traffic Management Research and Development Seminar, 2011.

[7] Mayer R H, Zondervan D J, Crow B M, et al. Development and operational transition of the first PBN-enabled departure separation standard $[\mathrm{C}]$ Integrated Communication, Navigation, and Surveillance Conference. IEEE, 2015:1-14. 